\title{
Review
}

\section{Pro/con debate: In patients who are potential candidates for organ donation after cardiac death, starting medications and/or interventions for the sole purpose of making the organs more viable is an acceptable practice}

\author{
Jason Phua ${ }^{1,2}$, Tow Keang Lim¹,3, David A Zygunn,5,6 and Christopher J Doig4,6,7 \\ 1'Division of Respiratory and Critical Care Medicine, Department of Medicine, National University Hospital, 5 Lower Kent Ridge Road, \\ Singapore 119074, Singapore \\ ${ }^{2}$ Interdepartmental Division of Critical Care Medicine, University Health Network and Mount Sinai Hospitals, Toronto, Ontario, Canada \\ ${ }^{3}$ Department of Medicine, Yong Loo Lin School of Medicine, National University of Singapore, Singapore \\ ${ }^{4}$ Department of Critical Care Medicine, University of Calgary, Alberta, Canada \\ ${ }^{5}$ Department of Clinical Neurosciences, University of Calgary, Alberta, Canada \\ ${ }^{6}$ Department of Community Health Sciences, University of Calgary, Alberta, Canada \\ ${ }^{7}$ Department of Medicine, University of Calgary, Alberta, Canada
}

Corresponding author: Tow Keang Lim, mdclimtk@nus.edu.sg

Published: 17 April 2007

Critical Care 2007, 11:211 (doi:10.1186/cc5711)

This article is online at http://ccforum.com/content/11/2/211

(c) 2007 BioMed Central Ltd

\begin{abstract}
Several hospitals have been developing programmes for organ donation after cardiac death. Such programmes offer options for organ donation to patients who do not meet brain-death criteria but wish to donate their organs after withdrawal of life-support. These programmes also increase the available organ pool at a time when demand exceeds supply. Given that potential donors are managed in intensive care units, intensivists will be key components of these programmes. Donation after cardiac death clearly carries a number of important ethical issues with it. In the present issue of Critical Care two established groups debate the ethical acceptability of using medications/interventions in potential organ donors for the sole purpose of making the organs more viable. Such debates will be an increasingly common component of intensivists' future practice.
\end{abstract}

\section{The scenario}

You are an intensivist in an institution that performs solid organ transplantations. In an effort to provide patients and families with increased opportunities to donate their organs, the institution has recently developed a policy for donation after cardiac death (DCD). With the new DCD policy, organ donation is offered to patients and their families in a controlled setting when death occurs immediately following the withdrawal of life-support. Based on your understanding of organ donation, you are aware there are certain medications (for example, inotropes to maintain tissue perfusion) and certain management practices that may allow the donated organs to have better outcome. You wonder about the ethics of starting interventions that will have no benefit to the dying patient but will benefit the organs that are about to be donated.

\section{Pro: Antemortem interventions to improve organ viability in donation after cardiac death are acceptable}

Jason Phua and Tow Keang Lim

What medications and interventions are started in potential donors before death for the sole purpose of making the organs more viable in DCD, and how do they affect organ function?

Firstly, inotropes and vasopressors are crucial for the preservation of organ perfusion in patients in shock. The majority of potential donors are hypotensive before cardiac death [1], and hypotension worsens graft function [2]. Secondly, anticoagulants such as heparin decrease the risk of thrombosis after the circulatory arrest and the negative consequences on organ function. To maximize effectiveness, heparin should ideally be administered before death into an intact circulation for systemic distribution [3]. Experimental data

$\mathrm{DCD}=$ donation after cardiac death.. 
show preserved organ function with antemortem but not postmortem heparin administration [4,5]. Thirdly, vasodilators such as phentolamine may enhance organ blood flow and lower the incidence of delayed renal graft function [6]. More controversial practices are the administration of thrombolytics and antemortem cannulation in preparation for the administration of cold preservation solution.

Although rarely performed in Europe, these practices are endorsed by major American and Canadian transplantation and ethical guidelines [3,7-9]. Indeed, most American DCD centres consider heparin administration at the time of withdrawal of lifesustaining treatment as the current standard of care $[3,7]$.

The acceptability of these practices should be evaluated according to Beauchamp and Childress' four moral principles [10]. As far as beneficence is concerned, none of these practices benefit the donors, at least not physically, since they will die regardless of the treatment provided. Most of the opposition to these practices, however, stems from the second principle of nonmaleficence. There is concern that anticoagulants and thrombolytics may cause bleeding and that vasodilators may cause hypotension - and therefore hasten death. Nevertheless, there is no evidence that heparin leads to sufficient bleeding after the withdrawal of life- sustaining therapies to cause death [7]. The guidelines, however, do state that heparin should only be used in patients with low bleeding risks, and phentolamine should only be used in patients without significant hypotension $[3,8]$.

The most important moral principle in DCD, however, is arguably that of autonomy $[3,8]$. If the potential donor or his/her designate gives informed consent for these organpreserving measures with a clear understanding of their possible side effects, who are healthcare professionals to object? Critics point to the lack of large randomized controlled trials to validate these measures. As the data available are very suggestive, however, while we await these trials (which may never be performed) the onus is on us to institute these measures to prevent any organs from going to waste. This is all the more crucial when one considers the last moral principle of justice, and the fact that these organs are a scarce resource.

To conclude, we believe that starting certain medications and interventions such as inotropes, vasopressors, heparin and phentolamine in potential donors for the sole purpose of making the organs in DCD more viable is an acceptable practice, provided they are used in patients with a low risk for side effects and that informed consent is provided.

\section{Con: The intended unintended and the principle of double effect}

David A Zygun and Christopher J Doig

The ethical principle of the 'rule of double effect' provides moral justification for the provision of certain forms of care at the end of life that result in death [11]. A practical example of this principle is the use of narcotics for pain relief, although respiratory depression and death are potential consequences. Application of this principle requires all four conditions to be met: the act must not belong to a category of acts considered evil; the good effect (for the patient), and not the bad effect, must be intended; the bad effect must not be a means to the good effect; and there must be a proportionally good reason for bringing about the bad effect [11,12]. This principle has also been used to justify antemortem interventions in DCD, but do these interventions meet these necessary elements? No, they do not.

The benefit of DCD is primarily to organ recipients and society [13-15]. This is evident in the published literature, where the common justification for DCD is to increase the supply of organs. Furthermore, the organ most likely to be recovered in DCD is the kidney, which will result in significant cost savings to healthcare systems by removing a patient from dialysis. Although there are some data that families may gain benefit from DCD, such as avoidance of delayed regret for missing the opportunity to donate organs [16] or the desire that donation will ease their grief [17], these reasons are also not for the benefit of the patient.
Is there potential harm to the donor (a hastening of death as a primary consequence)? Antemortem intravenous heparin and phentolamine are two interventions often considered. Most DCD donors are individuals with neurological injury, and heparin poses more than a theoretical risk of precipitating or exacerbating intracranial haemorrhage and hastening death. Phentolamine, a potent vasodilator, may precariously decrease blood pressure and hasten haemodynamic collapse in any ICU patient, and would be particularly harmful in a patient with impaired cerebral autoregulation. There are other interventions that might also be considered, such as intravenous fluid administration to maintain urine output (would this be acceptable if the patient has concomitant hydrostatic pulmonary oedema?). Simply, none of these interventions would be reasonably provided outside the setting of DCD, all are credibly associated with potential harm to the dying patient, and the perceived benefit of DCD may be directly gained through the harm caused (a more rapid death).

The principle of 'double effect' is suggested as an appropriate ethical framework to support antemortem interventions in DCD donors. The requisite elements of this principle are not met, and this principle cannot be used as a moral justification. As such, antemortem interventions with a risk to harm the patient violate the moral duty to 'first do no harm', 
and should not be condoned. Finally, invoking the principle of double effect places this principle in jeopardy as a reasonable justification for many appropriate interventions in palliative care treatment; if this principle is brought into disrepute, it may be harmful to palliative care patients, society and the practice of medicine.

\section{Pro's response: Involve Beauchamp and Childress' moral principles, not the doctrine of double effect}

Jason Phua and Tow Keang Lim

Some argue that by facilitating a successful DCD such antemortem interventions benefit the donor by fulfilling his/her wishes, benefit the donor's family by easing their grief, and benefit the recipient $[3,18]$. Detractors denounce this interpretation of the doctrine of double effect as medical sophistry. We propose that instead of focusing on this doctrine, these interventions should be evaluated according to Beauchamp and Childress' moral principles [10].

We reiterate that any bad effects of heparin and phentolamine must not be exaggerated without medical evidence. These interventions benefit DCD by improving organ viability, not by causing 'a more rapid death'.

\section{Con's response: The four tenets - out of tune}

David A Zygun and Christopher J Doig

Violation of the principle of beneficence has been acknowledged. With nonmaleficence, absence of evidence of harm does not equal proof of absence of harm; the incidence of harm from heparin is credible and has not been systematically examined. A standard of practice to use heparin without good clinical evidence of benefit and lacking measurement of potential harm is not a credible argument. Most potential DCD candidates are not competent to consent. Families as proxy are not acting in the patient's autonomous interest in consenting to treatment that will not benefit and may harm the patient, irrespective of benefit of family or another third party (society, organ recipient). Importantly, the statement 'they will die regardless of the treatment' is not factual [19]. Finally, justice requires an equal share of not only benefits, but also of burden. Given premortem intervention requires the dying patient to solely bear the burden; justice cannot be elicited to support such interventions.

\section{Competing interests}

The authors declare that they have no competing interests.

\section{References}

1. Revelly JP, Imperatori L, Maravic P, Schaller MD, Chiolero R: Are terminally ill patients dying in the ICU suitable for non-heart beating organ donation? Intensive Care Med 2006, 32:708-712.

2. Whelchel JD, Diethelm AG, Phillips MD, Schein LG: The effect of high dose dopamine in cadaveric donor management on delayed graft function and graft survival following renal transplantation. Transplant Proc 1986, 18:523-527.

3. Steering Committee, Donation after Cardiocirculatory Death: A Canadian Forum: Donation after Cardiocirculatory Death: A Canadian Forum: Report and Recommendations. Edmonton: The Canadian Council for Donation and Transplantation; 2005.

4. Boglione MM, Morandini MA, Barrenechea ME, Rubio RA, Aguilar $D$ : Pre-arrest heparinization and ventilation during warm ischemia preserves lung function in non-heart-beating donors. J Pediatr Surg 1999, 34:1805-1809.

5. Richter S, Yamauchi J, Minor T, Menger MD, Vollmar B: Heparin/phentolamine does not improve kidney perfusion with HTK solution after prolonged warm ischemia in a rat nonheart-beating donor model. Transplant Proc 2000, 32:23-24.

6. Polyak MM, Arrington BO, Kapur S, Stubenbord WT, Kinkhabwala $\mathrm{M}$ : Donor treatment with phentolamine mesylate improves machine preservation dynamics and early renal allograft function. Transplantation 2000, 69:184-186.

7. Bernat JL, D'Alessandro AM, Port FK, Bleck TP, Heard SO, Medina J, Rosenbaum SH, Devita MA, Gaston RS, Merion RM, et al.: Report of a National Conference on Donation after cardiac death. Am J Transplant 2006, 6:281-291.

8. Ethics Committee, American College of Critical Care Medicine, Society of Critical Care Medicine: Recommendations for nonheartbeating organ donation. A position paper by the Ethics Committee, American College of Critical Care Medicine, Society of Critical Care Medicine. Crit Care Med 2001, 29:1826-1831.

9. Institute of Medicine: Non-heart-beating Organ Transplantation: Practice and Protocols. Washington, DC: National Academy Press; 2000

10. Beauchamp TL, Childress JF: Principles of Biomedical Ethics. 5th edn. New York: Oxford University Press; 2001.

11. Quill TE, Dresser R, Brock DW: The rule of double effect - a critique of its role in end-of-life decision making. N Engl J Med 1997, 337:1768-1771.

12. Hawryluck LA, Harvey WR: Analgesia, virtue, and the principle of double effect. J Palliat Care 2000, 16 (Suppl):S24-S30.

13. Campbell GM, Sutherland FR: Non-heart-beating organ donors as a source of kidneys for transplantation: a chart review. CMAJ 1999, 160:1573-1576.

14. Kowalski AE, Light JA, Ritchie WO, Sasaki TM, Callender CO, Gage F: A new approach for increasing the organ supply. Clin Transplant 1996, 10:653-657.

15. Whiting JF, Kiberd B, Kalo Z, Keown P, Roels L, Kjerulf M: Costeffectiveness of organ donation: evaluating investment into donor action and other donor initiatives. Am J Transplant 2004, 4:569-573.

16. Oliver RC, Sturtevant JP, Scheetz JP, Fallat ME: Beneficial effects of a hospital bereavement intervention program after 
traumatic childhood death. J Trauma 2001, 50:440-446; discussion 447-448.

17. Bellali T, Papadatou D: Parental grief following the brain death of a child: does consent or refusal to organ donation affect their grief? Death Stud 2006, 30:883-917.

18. Veatch RM: Transplantation Ethics. Washington, DC: Georgetown University Press; 2000.

19. Cook D, Rocker G, Marshall J, Sjokvist P, Dodek P, Griffith L, Freitag A, Varon J, Bradley C, Levy M, et al.: Withdrawal of mechanical ventilation in anticipation of death in the intensive care unit. N Engl J Med 2003, 349:1123-1132. 